\title{
Packing Confined Hard Spheres Denser with Adaptive Prism Phases
}

\author{
Erdal C. Oğuz, ${ }^{1, *}$ Matthieu Marechal, ${ }^{1}$ Fernando Ramiro-Manzano, ${ }^{2}$ Isabelle Rodriguez, ${ }^{3,4}$ René Messina, ${ }^{1,5}$ \\ Francisco J. Meseguer, ${ }^{3,4}$ and Hartmut Löwen ${ }^{1}$ \\ ${ }^{1}$ Institut für Theoretische Physik II: Weiche Materie, Heinrich-Heine-Universität Düsseldorf, \\ Universitätsstraße 1, 40225 Düsseldorf, Germany \\ ${ }^{2}$ Nanoscience Laboratory, Department Physics, University of Trento, Via Sommarive 14, I-38050 Trento, Italy \\ ${ }^{3}$ Centro de Tecnologias Fisicas, Unidad Asociada ICMM/CSIC-UPV, Universidad Politécnica de Valencia, \\ Avenida Los Naranjos s/n, 46022 Valencia, Spain \\ ${ }^{4}$ Instituto de Ciencia de Materiales de Madrid CSIC, 28049 Madrid, Spain \\ ${ }^{5}$ Institut de Chimie, Physique et Matériaux (ICPM), Université de Lorraine, 1 Boulevard Arago, 57078 Metz-Cedex 3, France
}

(Received 27 September 2012; published 21 November 2012)

\begin{abstract}
We show that hard spheres confined between two parallel hard plates pack denser with periodic adaptive prismatic structures which are composed of alternating prisms of spheres. The internal structure of the prisms adapts to the slit height which results in close packings for a range of plate separations, just above the distance where three intersecting square layers fit exactly between the plates. The adaptive prism phases are also observed in real-space experiments on confined sterically stabilized colloids and in Monte Carlo simulations at finite pressure.
\end{abstract}

DOI: 10.1103/PhysRevLett.109.218301

PACS numbers: 82.70.Dd, 05.20.Jj, 64.70.K-, 68.65.Ac

How to pack the largest number of hard objects in a given volume is a classic optimization problem in pure geometry [1]. The close-packed structures obtained from such optimizations are also pivotal in understanding the basic physical mechanisms behind freezing [2,3] and glass formation [4]. Moreover, close-packed structures are highly relevant to numerous applications ranging from packaging macroscopic bodies and granulates [5] to the self-assembly of colloidal [6] and biological [7,8] soft matter. For the case of hard spheres, Kepler conjectured that the highest-packing density should be that of a periodic face-centered-cubic (fcc) lattice composed of stacked hexagonal layers; it took until 2005 for a strict mathematical proof [9]. More recent studies on close packing concern either nonspherical hard objects [10] such as ellipsoids $[11,12]$, convex polyhedra $[13,14]$ (in particular tetrahedra [15]), and irregular nonconvex bodies [16] or hard spheres confined in hard containers [17-19] or other complex environments.

If hard spheres of diameter $\sigma$ are confined between two hard parallel plates of distance $H$, as schematically illustrated in Fig. 1, the close-packed volume fraction $\phi$ and its associated structure depend on the ratio $H / \sigma$. Typically, the complexity of the observed phases increases tremendously on confining the system. Parallel slices from the fcc bulk crystal are only close packed for certain values of $H / \sigma$ : A stack of $n$ hexagonal (square) layers aligned with the walls, denoted by $n \triangle(n \square)$, is best packed at the plate separation $H_{n \triangle}\left(H_{n \square}\right)$ where the layers exactly fit between the walls. Clearly, for the minimal plate distance $H \equiv$ $H_{1 \triangle}=\sigma$, packing by a hexagonal monolayer is optimal. Increasing $H / \sigma$ up to $H_{2 \triangle}$, a buckled monolayer [20] and then a rhombic bilayer [21] become close packed.
However, for $H_{2 \triangle}<H<H_{4 \triangle}$, the close-packed structures are much more complex and still debated. Both, prism phases with alternating parallel prismlike arrays composed of hexagonal and square base $[22,23]$ and morphologies derived from the hexagonal-close-packed (hcp) structure $[24,25]$ were proposed as possible candidates.

For confined hard spheres, the knowledge and control over the close-packed configuration is of central relevance for at least two reasons. First, the hard sphere system away from close-packing is of fundamental interest as a quasitwo-dimensional statistical mechanics model. At low densities, a hard sphere gas is stable, which will crystallize as the density is increased beyond some threshold value. As such, the model represents a classical route to understand freezing between two and three spatial dimensions [26]. The associated fluid-solid transition will be strongly affected by the close-packed structure. Second, the confined hard sphere model is almost perfectly realized in nature by mesoscopic sterically stabilized colloidal suspensions [22,27] which can be confined between glass plates providing a slitlike confinement. At high imposed pressures, colloids will self-assemble into the close-packed structures. It has been shown that this is the key for the

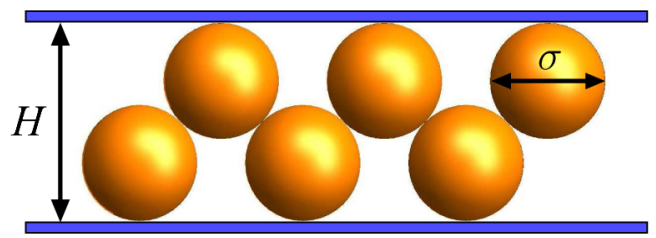

FIG. 1 (color online). Schematic illustration of hard spheres of diameter $\sigma$ confined between two parallel hard plates of separation $H$. 
controlled fabrication of nanosieves and of membranes with desired morphology [28].

In this Letter, we explore the close-packed structures of confined hard spheres by combining numerical optimization, experiments and computer simulation. Using a systematic penalty optimization method, we find the whole cascade of close-packed structures in the range of plate distances $H_{1 \triangle}<H<H_{4 \triangle}$. As an important building block for close packing, an adaptive prism is identified which adjusts its internal structure flexibly to the slit height $H / \sigma$. This prism has a base with a rhombic symmetry and neighboring prismatic arrays are shifted relative to each other. The resulting adaptive structure maximizes the packing fraction in the regime beyond $H_{3 \square}$. We also propose a further close-packing prism phase of square symmetry that packs densest in the regime just beyond $H_{4 \square}$ and shows a two-dimensional relative lateral shift between the prisms. We confirm the stability of the new adaptive prismatic structures both in real-space experiments on confined sterically stabilized colloids and in Monte Carlo simulations at finite pressure. In the following, we first describe the results from the penalty method, then discuss real-space data for confined colloidal samples and subsequently turn to Monte Carlo simulation results. Details of the numerical methods, experiments, and simulations are listed in the Supplemental Material [29].

In our numerical calculations, we consider periodic structures with up to 12 particles per unit cell thereby covering all hitherto proposed structures [29]. To maximize the packing fraction $\phi$, we optimized the cell shape and the particle coordinates of these structures. However, investigating the dense packing of hard spheres accommodates a constrained optimization: the free volume must be minimized under the constraint of nonoverlapping spheres. To circumvent the discontinuous, constrained optimization, we employed the penalty method [30] in our numerical calculations. By adding a penalty term in case the spheres intersect which depends continuously on the overlap volume, we obtained a continuous and unconstrained penalty function which can be minimized in the classic way to predict the optimal particle coordinates. The penalty method offers the flexibility to use a relatively broad range of candidate crystalline lattices and has recently been shown to allow a very efficient handling of packing problems [31].

The resulting volume fractions of the densest packed phases are shown in Fig. 2 as a function of $H / \sigma$ in the regime between the hexagonal monolayer $1 \triangle(H / \sigma=1)$ and the triangular tetralayer $4 \triangle(H / \sigma=\sqrt{6}+1)$. For $H_{1 \triangle}<H<H_{2 \triangle}$ the classic sequence [20,21,32] $1 \triangle \rightarrow$ $B \rightarrow 2 \square \rightarrow 2 R \rightarrow 2 \triangle$ is confirmed. Here, $B$ is a buckled hexagonal layer with rectangular symmetry and the $2 R$ crystal consists of two staggered rhombic layers.

For $H_{2 \triangle}<H<H_{4 \triangle}$, there is a much more complex cascade of close-packed structures. In the transition regime

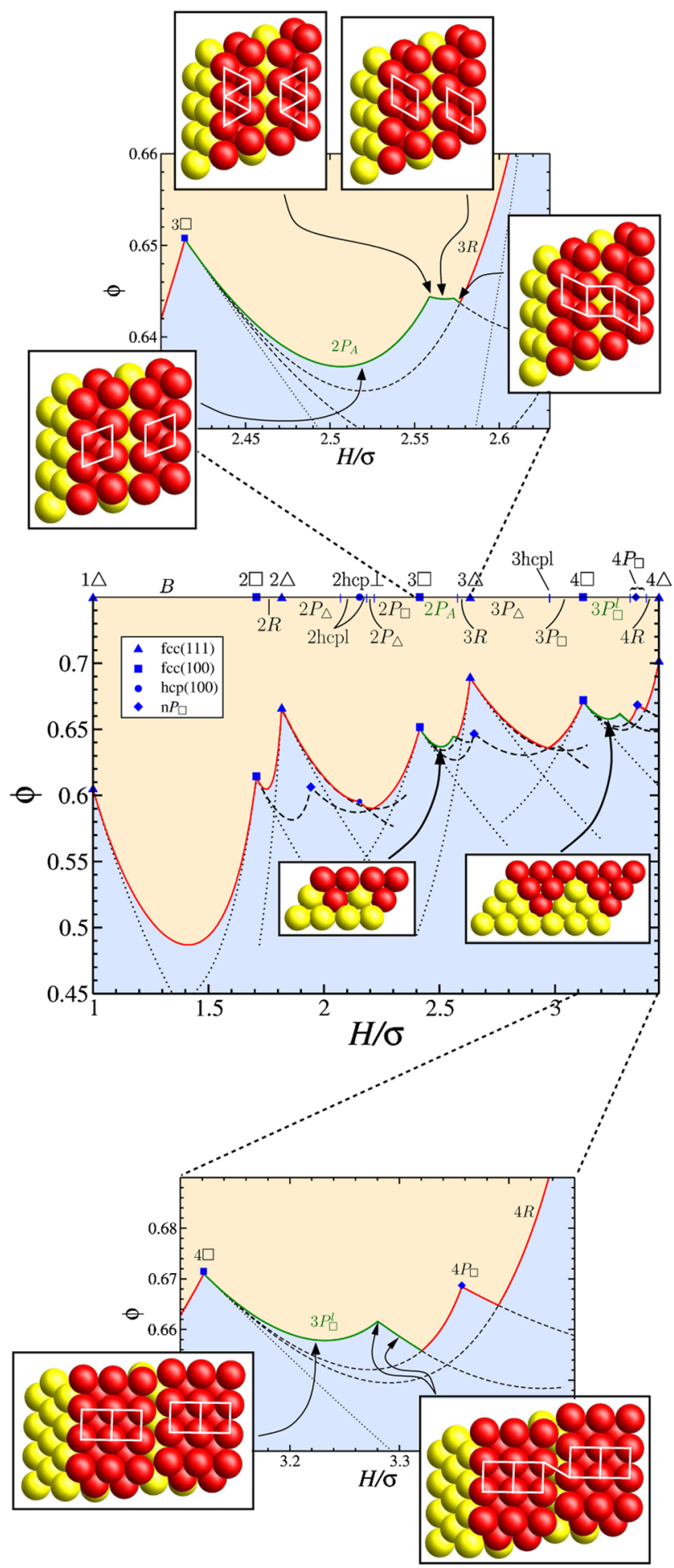

FIG. 2 (color online). Packing fraction $\phi$ versus dimensionless height $H / \sigma$. The best-packing phases are indicated by symbols on the top axis of the middle panel and their packing fractions are shown as the full lines. Dashed and dotted lines denote the nonclose packed $n \square$ [fcc(100)], $n \triangle$ [fcc(111)], $n \mathrm{hcpl}, n P_{\triangle}$, and $n P_{\square}$. The top and bottom panel show enlargements of the regions where the new prism phases were found. Side views (middle panel) and top views (top and bottom panels) show the structure of these phases, where white lines denote bonds between touching particles. 
$n \triangle \rightarrow(n+1) \square$ for $n=2,3$, on the one hand, we recover all of the phases found previously. Here, we obtain the sequence $2 \triangle \rightarrow 2 P_{\triangle} \rightarrow 2 \mathrm{hcpl} \rightarrow 2 \mathrm{hcp} \perp \rightarrow 2 \mathrm{hcpl} \rightarrow 2 P_{\square} \rightarrow$ $3 \square$ and $3 \triangle \rightarrow 3 P_{\triangle} \rightarrow 3 \mathrm{hcpl} \rightarrow 2 P_{\square} \rightarrow 3 \square$, where the following phases are encountered: The $2 n$-layered phases $n P_{\triangle}$ and $n P_{\square}$ consist of alternating prismlike densepacked $n$-layered arrays of spheres with triangular $(\triangle)$ and square $(\square)$ basis shapes $([22,23,27])$. Moreover, the $2 n$-layered phases $n$ hcp $\perp$ [33] and $n$ hcp-like with rectangular symmetry (see Refs. [24,25]) are found. For $n=3$, however, the $n$ hcp-like phase is only close packed in a tiny regime, whereas $n$ hcp $\perp$ is not found at all (see Fig. 2).

In the range $3 \square \rightarrow 3 \triangle$, the new adaptive prism phase $2 P_{A}$ is predicted to be close packed. Representative intralayer touching bonds are indicated by white lines in Fig. 2 (upper and lower panel) to underline the symmetry of the corresponding prismatic structure. The $2 P_{A}$ phase adapts its internal structure flexibly to an increase of the slit width $H$. In fact, the symmetry of its prism blocks is rhombic which spans the whole range between the square symmetry of the underlying phase $3 \square$ and the triangular base (see white lines in Fig. 2, upper panel). Likewise, we noticed the stability of the $3 P_{\square}^{l}$ prism phase with square basis shape (cf. Fig. 2, lower panel) in the transition regime $4 \square \rightarrow 4 \triangle$ whose prisms exhibit a longitudinal shift (i.e., in the lengthwise direction of the prisms) in addition to the usual shift perpendicular to the lengthwise direction of the prisms. Finally, the other densest packed phases are multilayered rhombic phases $3 R$ and $4 R$ as well as a square prism phase $4 P_{\square}$, see Fig. 2 .

To verify our theoretical results, we performed real-space experiments with nanometer-sized colloids. We employed Polystyrene particles with diameters $\sigma$ in the range from 245 to $800 \mathrm{~nm}$ (Ikerlat Polymers) to study certain $\mathrm{H} / \sigma$ values. We created a confining wedge cell with a very small opening angle $\left(10^{-4} \mathrm{rad}\right)$ and slit height $H<6 \mu \mathrm{m}$ (see Ref. [29]). The varying slight height inherent to the wedge geometry allows many transitions between different crystals in the same cell. Finally, after the sample was dried, we detached the Polystyrene covering plate. Some particles stuck to the covering plate during its removal resulting in holes in the top layer of particles, which allowed us to study the structure in the layers below. We recorded Scanning Electron Microscope (SEM) images from the top facets and side edges by cleaving the samples or by focused ion beam milling following the crystal planes.

Concentrating on the regime $3 \square \rightarrow 3 \triangle$, we found evidence of the adaptive $2 P_{A}$ phase. Also, the $3 P_{\square}^{l}$ phase has been observed for larger plate separation distances. As an example, SEM images of $2 P_{A}$ and $3 P_{\square}^{l}$ are shown in Fig. 3 along with a simulation snapshot for comparison.

Experiments on colloidal systems, such as ours, are necessarily performed at finite pressure. In order to investigate the stability of the new prism phases away from close packing, we performed Monte Carlo simulations at a fixed
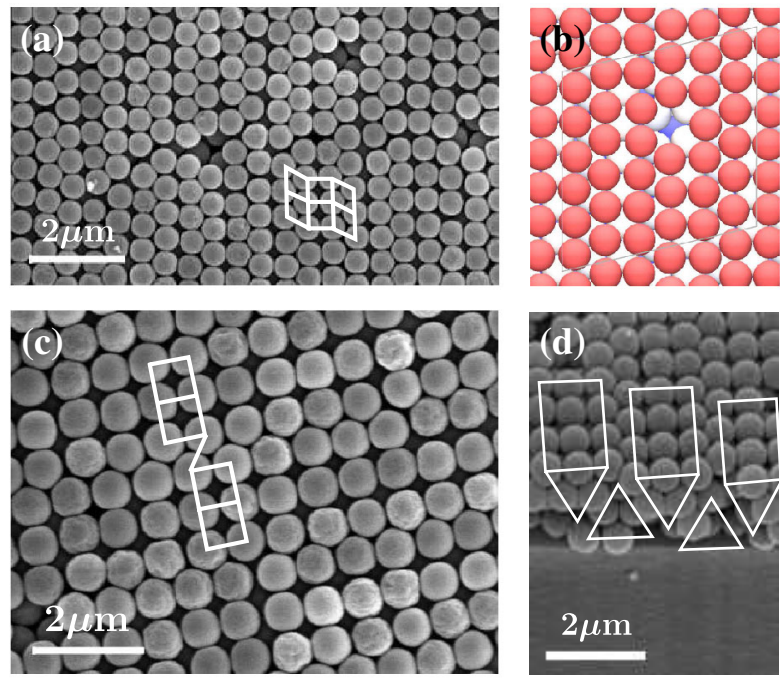

FIG. 3 (color online). SEM micrographs of the prism phase found in this work: $2 P_{A}$ (a) and $3 P_{\square}^{l}$ (c),(d). A few particles were removed from the top layer upon detachment of the covering plate allowing access to the structure in the layer below. A simulation snapshot, where a particle was also removed (after the simulation), is shown in (b). White lines indicate the symmetry of each phase (a),(c) as well as the structure of the prism arrays in the side view of $3 P_{\square}^{l}$ (d).

lateral pressure $P_{l}=-H^{-1} \partial F / \partial A$, where $F$ is the free energy and $A$ denotes the area of the system. This definition of pressure is such that it approaches the bulk pressure as $H$ increases. The discovery of new crystal phases in this and previous theoretical works at infinite pressure after the previous simulation work that addressed the stability at finite pressure begs the question how stable these phases are at a high, but finite pressure [34]. We simulated the system at a high pressure $P_{l} \sigma^{3} / k_{B} T=40$, for which the system would equilibrate within a reasonable time (for comparison the bulk crystallization pressure is $P \sigma^{3} / k_{B} T=11.56$ [35]). The success of cell theoryeffectively a single-particle theory-at high densities indicates that phase behavior at high pressures can be accurately modeled using relatively small systems. Our variable-shape simulation box contained $m \times m \times n$ particles, where $n$ is the number of layers and $4 \leq m \leq 8$ [29]. In Fig. 4, we compare finite-pressure simulation data to theoretical results at infinite pressure. We clearly see that the packing fractions in both cases feature a qualitatively similar course. However, some phases vanish for finite pressure as this regime is dominated by broadened stability regimes of $n \triangle$ and $n \square$ phases. In detail, the $2 R, 3 R$, $2 \mathrm{hcp} \perp$, 3hcpl, $2 P_{\triangle}$, and $3 P_{\triangle}$ phases are not found for the finite pressure and accuracy $H / \sigma \pm 0.025$ chosen in the simulations. As can be further seen, the adaptive prism phase $2 P_{A}$ and $3 P_{\square}^{l}$ found in this Letter are stable at this pressure and, therefore, also at all higher pressures.

These simulations help explain the absence of the triangular prism phase in the experiments (see Ref. [27]). 


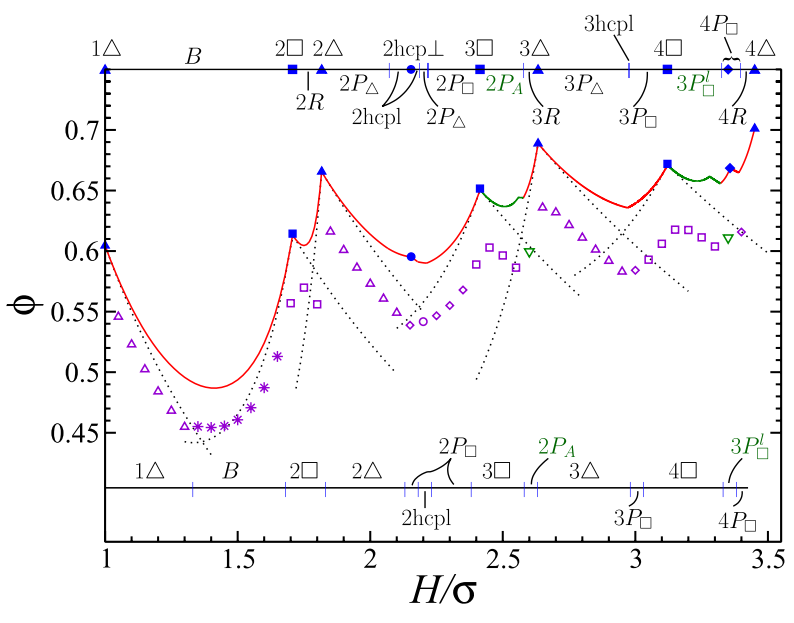

FIG. 4 (color online). Isobaric packing fractions $\phi$, as measured in simulations at fixed lateral pressure $P_{l} \sigma^{3} / k_{B} T=40$ for confined hard spheres, versus dimensionless separation $H / \sigma$ (empty symbols) compared to the theoretical results of Fig. 2 (lines). Each type of empty symbol denotes a different phase. The observed phases are indicated by the symbols on the horizontal lines at the top (theory) and bottom (simulation) of the graph.

We also performed simulations with the triangular prism phases as initial configuration. At the values for $H$ where the triangular prism phase has the highest density of all possible phases, the $n P_{\triangle}$ phase appears to consist of $n$ only slightly distorted hexagonal layers. At finite pressure, the small distortions can quickly disappear and a regular triangular crystal can be formed. This is a typical scenario for crystal-crystal transitions for hard particles, where the close-packed crystal phase transforms into a highersymmetry crystal with a slightly lower density, but a greater entropy, as the pressure is decreased sufficiently. At larger values of $H$ than those investigated here, the triangular prisms are significantly enhanced and do not transform so easily to triangular crystals (cf. Ref. [23]). Preliminary simulations show that indeed stable triangular prism phases can be found for larger values for $H$ (see Ref. [29]).

In conclusion, we explored the close-packed structures of hard spheres confined between hard plates in a broad range of plate separations by combining theory, experiment, and simulation. We identified adaptive prism phases with rhombic symmetry which pack densest in certain ranges of the slit width. An adaptive prism phase optimizes packing by adjusting its base symmetry flexibly to the slit width. Also, we showed a high persistence of these adaptive prism phases at finite, but large pressure, using experiments and simulations. We anticipate that the adaptive prism phase will play a key role for even higher plate distances, $H / \sigma>3.5$, as ideal interpolating close-packed building blocks.

The adaptive prism phases found here offer new opportunities for several applications. For example, the reported structures possess pronounced symmetry directions whose alignment can be internally controlled by the slit height instead of using external fields (e.g., electric fields, cf. Ref. [36]). As a consequence, these phases can serve as switchable materials. Furthermore, we expect an unusual and anisotropic dynamical response of the multilayered prism phases upon shear [37] with possibly molten grain boundaries which can be exploited to tune the rheological properties of thin crystalline sheets. Finally, by varying $H$, it is possible to tune the whole complex cascade of close-packed structures. This may be of importance to fabricate nanosieves or porous membranes [28] in a controlled way.

We thank Elvira Bonet, Moisés Garín, and Kevin Mutch for helpful discussions. This work was partially supported by the DFG within the SFB TR6 (Project D1), and by the Spanish CICyT Projects FIS2009-07812 and PROMETEO/2010/043. F. R.-M. acknowledges the support from the EU Marie Curie Project APPCOPTOR275150 (FP7-PEOPLE-2010-IEF).

*ecoguz@ thphy.uni-duesseldorf.de; http://www2.thphy .uni-duesseldorf.de/ ecoguz/

[1] T. C. Hales, Discrete Comput. Geom. 36, 5 (2006).

[2] J. D. Bernal, Nature (London) 185, 68 (1960).

[3] F.H. Stillinger, E. A. DiMarzio, and R. L. Kornegay, J. Chem. Phys. 40, 1564 (1964).

[4] R. Zallen, The Physics of Amorphous Solids (Wiley, New York, 1983).

[5] S.F. Edwards, Granular Matter (Springer-Verlag, New York, 1994).

[6] W. B. Russel, D. A. Saville, and W. R. Schowalter, Colloidal Dispersions (Cambridge University Press, Cambridge, England, 1989).

[7] J. Liang and K. A. Dill, Biophys. J. 81, 751 (2001).

[8] P. K. Purohit, J. Kondev, and R. Phillips, Proc. Natl. Acad. Sci. U.S.A. 100, 3173 (2003).

[9] T. C. Hales, Ann. Math. 162, 1065 (2005).

[10] For a recent review, see, e.g., S. Torquato and F. H. Stillinger, Rev. Mod. Phys. 82, 2633 (2010).

[11] A. Donev, F. H. Stillinger, P. M. Chaikin, and S. Torquato, Phys. Rev. Lett. 92, 255506 (2004).

[12] S. Sacanna, L. Rossi, A. Wouterse, and A. P. Philipse, J. Phys. Condens. Matter 19, 376108 (2007).

[13] P. F. Damasceno, M. Engel, and S. C. Glotzer, Science 337, 453 (2012).

[14] S. Torquato, Nature (London) 460, 876 (2009).

[15] A. Haji-Akbari, M. Engel, A. S. Keys, X. Zheng, R. G. Petschek, P. Palffy-Muhoray, and S.C. Glotzer, Nature (London) 462, 773 (2009).

[16] J. de Graaf, R. van Roij, and M. Dijkstra, Phys. Rev. Lett. 107, 155501 (2011).

[17] G. T. Pickett, M. Gross, and H. Okuyama, Phys. Rev. Lett. 85, 3652 (2000).

[18] A. Mughal, H. K. Chan, and D. Weaire, Phys. Rev. Lett. 106, 115704 (2011). 
[19] R.P.A. Dullens, D. G. A. L. Aarts, W. K. Kegel, and H. N.W. Lekkerkerker, Mol. Phys. 103, 3195 (2005).

[20] P. Pieranski, L. Strzelecki, and B. Pansu, Phys. Rev. Lett. 50, 900 (1983).

[21] M. Schmidt and H. Löwen, Phys. Rev. Lett. 76, 4552 (1996); M. Schmidt and H. Löwen, Phys. Rev. E 55, 7228 (1997).

[22] S. Neser, C. Bechinger, P. Leiderer, and T. Palberg, Phys. Rev. Lett. 79, 2348 (1997).

[23] A. Fortini and M. Dijkstra, J. Phys. Condens. Matter 18, L371 (2006).

[24] F. Ramiro-Manzano, E. Bonet, I. Rodriguez, and F. Meseguer, Phys. Rev. E 76, 050401(R) (2007).

[25] A. B. Fontecha, T. Palberg, and H. J. Schöpe, Phys. Rev. E 76, 050402(R) (2007).

[26] Y. Peng, Z. R. Wang, A. M. Alsayed, A. G. Yodh, and Y. Han, Phys. Rev. E 83, 011404 (2011).

[27] F. Ramiro-Manzano, E. Bonet, I. Rodriguez, and F. Meseguer, Soft Matter 5, 4279 (2009).

[28] F. Yan and W. A. Goedel, Chem. Mater. 16, 1622 (2004).
[29] See Supplemental Material at http://link.aps.org/ supplemental/10.1103/PhysRevLett.109.218301 for technical details and depictions of triangular prism and degenerated rhombic phases.

[30] A. V. Fiacco and G.P. McCormick, Nonlinear Programming: Sequential Unconstrained Minimization Techniques (Wiley, New York, 1968).

[31] L. Assoud and R. Messina, Phys. Rev. E 83, 036113 (2011).

[32] S. Grandner and S.H.L. Klapp, J. Chem. Phys. 129, 244703 (2008).

[33] This phase structure corresponds to the (100) plane of the hexagonal close-packed solid and, therefore, it is referred to as $n$ hcp(100) in Ref. [24].

[34] K. Franzrahe and P. Nielaba, Phys. Rev. E 79, 051505 (2009).

[35] T. Zykova-Timan, J. Horbach, and K. Binder, J. Chem. Phys. 133, 014705 (2010).

[36] A.P. Bartlett, A. K. Agarwal, and A. Yethiraj, Langmuir 27, 4313 (2011).

[37] I. Cohen, T. G. Mason, and D. A. Weitz, Phys. Rev. Lett. 93, 046001 (2004). 